\title{
ON THE MINIMIZERS OF THE FUSION FRAME POTENTIAL
}

\author{
SIGRID B. HEINEKEN ${ }^{1, *}$, JUAN P. LLARENA ${ }^{2}$, AND PATRICIA M. MORILLAS ${ }^{2}$ \\ ${ }^{1}$ Departamento de Matemática, FCEyN, Universidad de Buenos Aires, Pabellón I, Ciudad \\ Universitaria, IMAS, UBA-CONICET, C1428EGA C.A.B.A. Buenos Aires, Argentina \\ 2 Instituto de Matemática Aplicada San Luis, UNSL-CONICET and Departamento de \\ Matemática, FCFMyN, UNSL, Ejército de los Andes 950, 5700 San Luis, Argentina
}

\begin{abstract}
We study the minimizers of the fusion frame potential in the case that both the weights and the dimensions of the subspaces are fixed and not necessarily equal. Using a concept of irregularity we provide a description of the local (that are also global) minimizers which projections are eigenoperators of the fusion frame operator. This result will be related to the existence of tight fusion frames. In this way we generalize results known for the classical vector frame potential.
\end{abstract}

Key words: Frames, Fusion frames, Fusion frame potential, Tight fusion frames, Fundamental inequality, Irregularity.

AMS subject classification: Primary 42C15; Secondary 42C99, 42C40, $15 \mathrm{~A} 60$.

\section{Introduction}

Fusion frames (or frames of subspaces) for a separable Hilbert space $\mathcal{H}$ are collections of closed subspaces and weights, that allow the reconstruction of each element of $\mathcal{H}$ from packets of coefficients (see [7, 8] and also [6, Chapter 13]). They are a generalization of frames [3, 6, 9, 10, and are useful in situations where a distributed processing by combining locally data vectors has to be implemented, such as distributing sensing, parallel processing and packet encoding.

In the present paper we consider the finite-dimensional case. Finite-dimensional Hilbert spaces and finite fusion frames [6] are an important tool in applications since they prevent the approximation problems that appear with the truncations in the infinite-dimensional case.

The concept of frame potential has been introduced by Benedetto and Fickus in 11. There the authors give a complete description of the structure of the unit-norm minimizers of this potential, and then characterize the existence of normalized tight frames in terms of these minimizers. This frame potential was also considered in [5] where the results of [1] are extended to the non-uniform length setting.

In 4, Casazza and Fickus introduced and studied the notion of fusion frame potential. This potential has been also extensively studied in [11. In this paper we use a different approach. We generalize results of [5] to the setting of fusion frames.

\footnotetext{
* Corresponding author. E-mail addresses: sheinek@dm.uba.ar (S. B. Heineken), jllarena@unsl.edu.ar (J. P. Llarena), morillas@unsl.edu.ar (P. M. Morillas).
} 
We study the minimizers of the fusion frame potential in the case that both the weights and the dimensions of the subspaces are fixed and not necessarily equal.

The organization of the paper is as follows. In section 2 we review concepts about frames, fusion frames and fusion frame potential. In Section 3 and 4, we provide a description of the local minimizers which projections are eigenoperators of the fusion frame operator. This description is completed in Section 5 using a concept of irregularity and it is shown that these local minimizers are also global. In section 6 , we relate the study of the minimizers of the fusion frame potential to the existence of tight fusion frames.

\section{Preliminaries}

In this section we recall the concepts of frame [3, 6, 9, 10, fusion frame 7, 8, (see also [6, Chapter 13]) and fusion frame potential [4, 11. We refer to the mentioned works for more details. We begin introducing some notation.

2.1. Notation. Let $\mathbb{F}=\mathbb{R}$ or $\mathbb{F}=\mathbb{C}$. Let $d, n \in \mathbb{N}$. Noting that any finite dimensional Hilbert space over $\mathbb{F}$ of dimension $d$ is isomorphic to $\mathbb{F}^{d}$ with the usual inner product we will work directly with this last Hilbert space.

We will identify the linear transformations from $\mathbb{F}^{d}$ to $\mathbb{F}^{n}$ with their matrix representation with respect to the standard bases of $\mathbb{F}^{d}$ and $\mathbb{F}^{n} . \mathbb{F}^{d \times n}$ denotes the set of matrices of order $d \times n$ with entries in $\mathbb{F}$. If $M \in \mathbb{F}^{d \times n}$, then $R(M)$ and $M^{*}$ denotes the range and the conjugate transpose of $M$, respectively. The elements of $\mathbb{F}^{n}$ will be considered as column vectors, i.e., we identify $\mathbb{F}^{n}$ with $\mathbb{F}^{n \times 1}$, and if $f \in \mathbb{F}^{n}$ then $f(i)$ denotes the $i$ th component of $f$. Let $M \in \mathbb{F}^{d \times n}$. We denote the entry $i, j$ of $M$ with $M(i, j)$ and the $j$ th column by $M(:, j)$. The inner product and the norm in $\mathbb{F}^{d}$ will be denoted by $\langle.,$.$\rangle and \|$.$\| , respectively. If T \in \mathbb{F}^{d \times n}$, then $\|T\|_{F}$ denotes the Frobenius norm of $T$.

The set of subspaces of $\mathbb{F}^{d}$ will be denoted by $\mathcal{S}$. If $V \in \mathcal{S}, \pi_{V} \in \mathbb{F}^{d \times d}$ denotes the orthogonal projection onto $V$.

\subsection{Frames and fusion frames.}

Definition 2.1. Let $\mathcal{F}=\left\{f_{k}\right\}_{k=1}^{K} \subset \mathbb{F}^{d}$.

(1) $\mathcal{F}$ is a frame for $\mathbb{F}^{d}$ if span $\mathcal{F}=\mathbb{F}^{d}$.

(2) If $\mathcal{F}$ is a frame for $\mathbb{F}^{d}$,

$$
S_{\mathcal{F}}: \mathbb{F}^{d} \rightarrow \mathbb{F}^{d}, S_{\mathcal{F}} f=\sum_{k=1}^{K}\left\langle f, f_{k}\right\rangle f_{k},
$$

is the frame operator of $\mathcal{F}$.

(3) $\mathcal{F}$ is an $\alpha$-tight frame, if $S_{\mathcal{F}}=\alpha I$. If $S_{\mathcal{F}}=I, \mathcal{F}$ is a Parseval frame.

If $\mathcal{F}$ is an $\alpha$-tight frame we have the following reconstruction formula

$$
f=\frac{1}{\alpha} \sum_{k=1}^{K}\left\langle f, f_{k}\right\rangle f_{k}, \text { for all } f \in \mathbb{F}^{d},
$$

where the scalar coefficients $\left\langle f, f_{k}\right\rangle, k=1, \ldots, K$, can be thought as a measure of the projection of $f$ onto each frame vector.

Fusion frames generalize the notion of frames. Each $f \in \mathbb{F}^{d}$ can be a represented via fusion frames is given by projections onto multidimensional subspaces. Before defining them we introduce the following concept. 
Definition 2.2. Let $\left\{W_{k}\right\}_{k=1}^{K}$ be a family of subspaces of $\mathbb{F}^{d}$, and let $\left\{w_{k}\right\}_{k=1}^{K}$ be a family of weights, i.e., $w_{k}>0$ for $k=1, \ldots, K$. Then $\left\{\left(W_{k}, w_{k}\right)\right\}_{k=1}^{K}$ is called a Bessel fusion sequence for $\mathbb{F}^{d}$.

Throughout the article we will assume that $K$ is fixed and we denote $\left\{W_{k}\right\}_{k=1}^{K} \subset$ $\mathcal{S}$ with $\mathbf{W},\left\{w_{k}\right\}_{k=1}^{K}$ with $\mathbf{w}$ and $\left\{\left(W_{k}, w_{k}\right)\right\}_{k=1}^{K}$ with $(\mathbf{W}, \mathbf{w})$. If $w_{1}=\cdots=w_{K}=w$ we write $w$ instead of $\mathbf{w}$. The set of Bessel fusion sequences in $\mathbb{F}^{d}$ with $K$ subspaces of $\mathbb{F}^{d}$ and weights will be denoted with $\mathcal{B}_{K}$.

Let $\mathcal{W}:=\bigoplus_{k=1}^{K} W_{k}=\left\{\left(f_{k}\right)_{k=1}^{K}: f_{k} \in W_{k}\right\}$ be the Hilbert space with

$$
\left\langle\left(f_{k}\right)_{k=1}^{K},\left(g_{k}\right)_{k=1}^{K}\right\rangle=\sum_{k=1}^{K}\left\langle f_{k}, g_{k}\right\rangle .
$$

Definition 2.3. Let $(\mathbf{W}, \mathbf{w}) \in \mathcal{B}_{K}$.

(1) $(\mathbf{W}, \mathbf{w})$ is called a fusion frame for $\mathbb{F}^{d}$ if $\operatorname{span} \bigcup_{k=1}^{K} W_{k}=\mathbb{F}^{d}$.

(2) $(\mathbf{W}, \mathbf{w})$ is a Riesz fusion basis if $\mathbb{F}^{d}$ is the direct sum of the $W_{k}$, and $(\mathbf{W}, 1)$ an orthonormal fusion basis if $\mathbb{F}^{d}$ is the orthogonal sum of the $W_{k}$.

(3) If $(\mathbf{W}, \mathbf{w})$ is a fusion frame for $\mathbb{F}^{d}$, the operator

$$
S_{\mathbf{W}, \mathbf{w}}: \mathbb{F}^{d} \rightarrow \mathbb{F}^{d}, S_{\mathbf{W}, \mathbf{w}}(f)=\sum_{k=1}^{K} w_{k}^{2} \pi_{W_{k}}(f)
$$

is called the fusion frame operator of $(\mathbf{W}, \mathbf{w})$.

(4) A fusion frame $(\mathbf{W}, \mathbf{w})$ is called an $\alpha$-tight fusion frame if $S_{\mathbf{W}, \mathbf{w}}=\alpha I$. If $S_{\mathbf{W}, \mathbf{w}}=I$ we say that it is a Parseval fusion frame.

If $(\mathbf{W}, \mathbf{w})$ is an $\alpha$-tight fusion frame we have the following reconstruction formula

$$
f=\frac{1}{\alpha} \sum_{k=1}^{K} w_{k}^{2} \pi_{W_{k}}(f), \text { for all } f \in \mathbb{F}^{d} .
$$

2.3. The fusion frame potential. The set of sequences of $K$ subspaces of $\mathbb{F}^{d}$ will be denoted with $\mathcal{S}_{K}$. In what follows we assume that the weights $\mathbf{w}$ are fixed. Let $K \in \mathbb{N}$ and $\mathbf{L}=\left(L_{1}, \ldots, L_{K}\right) \in \mathbb{N}^{K}$. If $L_{1}=\cdots=L_{K}=L$ we write $L$ instead of L. We consider the set

$$
\mathcal{S}_{K}(\mathbf{L}):=\left\{\mathbf{W} \in \mathcal{S}_{K}: \operatorname{dim}\left(W_{k}\right)=L_{k} \text { for every } k \in\{1, \ldots, K\}\right\} .
$$

We define the distance between $W, V \in \mathcal{S}$ as

$$
d(W, V)=\left\|\pi_{W}-\pi_{V}\right\|_{F}
$$

and the distance between $\mathbf{W}, \mathbf{V} \in \mathcal{S}_{K}$ as

$$
d(\mathbf{W}, \mathbf{V})=\left[\sum_{k=1}^{K}\left\|\pi_{W_{k}}-\pi_{V_{k}}\right\|_{F}^{2}\right]^{1 / 2} .
$$

With this metric the set $\mathcal{S}_{K}(\mathbf{L})$ is compact (this follows from [11, Lemma 4.3.1]).

Following [4] we define the fusion frame potential $F F P_{\mathbf{w}}: \mathcal{S}_{K}(\mathbf{L}) \rightarrow \mathbb{R}$ as

$$
F F P_{\mathbf{w}}(\mathbf{W})=\operatorname{tr}\left(S_{\mathbf{W}, \mathbf{w}}^{2}\right) .
$$

Note that

$$
\operatorname{tr}\left(S_{\mathbf{W}, \mathbf{w}}^{2}\right)=\operatorname{tr}\left(\sum_{k=1}^{K} w_{k}^{2} \pi_{W_{k}} \sum_{k=1}^{K} w_{k}^{2} \pi_{W_{k}}\right)=\sum_{k, k^{\prime}=1}^{K} w_{k}^{2} w_{k \prime}^{2} \operatorname{tr}\left(\pi_{W_{k}} \pi_{W_{k^{\prime}}}\right)
$$

and each term in the above sum is a nonnegative real number since 


$$
\begin{aligned}
\operatorname{tr}\left(\pi_{W_{k}} \pi_{W_{k^{\prime}}}\right) & =\operatorname{tr}\left(\pi_{W_{k}}^{2} \pi_{W_{k^{\prime}}}^{2}\right)=\operatorname{tr}\left(\pi_{W_{k}} \pi_{W_{k^{\prime}}} \pi_{W_{k^{\prime}}} \pi_{W_{k}}\right)=\operatorname{tr}\left(\pi_{W_{k}}^{*} \pi_{W_{k^{\prime}}}^{*} \pi_{W_{k^{\prime}}} \pi_{W_{k}}\right) \\
& =\operatorname{tr}\left(\left(\pi_{W_{k^{\prime}}} \pi_{W_{k}}\right)^{*} \pi_{W_{k^{\prime}}} \pi_{W_{k}}\right)=\left\|\pi_{W_{k^{\prime}}} \pi_{W_{k}}\right\|_{F}^{2} .
\end{aligned}
$$

Analogous as in [4. Proposition 1] the following can be proved:

Proposition 2.4. If $\mathbf{W} \in \mathcal{S}_{K}(\mathbf{L})$, then

$$
F F P_{\mathbf{w}}(\mathbf{W}) \geq \frac{1}{d}\left(\sum_{k=1}^{K} w_{k}^{2} L_{k}\right)^{2},
$$

with equality holding in 2.4 if and only if $(\mathbf{W}, \mathbf{w})$ is a tight fusion frame for $\mathbb{F}^{d}$.

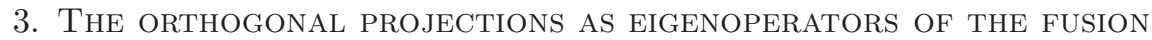 FRAME OPERATOR}

From now on we denote the different eigenvalues of $S_{\mathrm{W}, \mathrm{w}}$ listed in decreasing order with $\left\{\lambda_{j}\right\}_{j=1}^{J}$ and the corresponding sequence of eigenspaces with $\left\{E_{j}\right\}_{j=1}^{J}$. We consider the index sets

$$
I_{j}=\left\{k \in\{1, \ldots, K\}: S_{\mathbf{W}, \mathbf{w}} \pi_{W_{k}}=\lambda_{j} \pi_{W_{k}}\right\} .
$$

We define the class of Bessel fusion sequences for which the orthogonal projections are "eigenoperators" of the fusion frame operator:

$\mathcal{E}=\left\{(\mathbf{W}, \mathbf{w}) \in \mathcal{B}_{K}: \forall k \in\{1, \ldots, K\} \exists j \in\{1, \ldots, J\}\right.$ such that $\left.S_{\mathbf{W}, \mathbf{w}} \pi_{W_{k}}=\lambda_{j} \pi_{W_{k}}\right\}$.

For $L_{1}=\cdots=L_{K}=1$, it can be concluded from the vectorial case that every local minimizer of $F F P_{\mathbf{w}}: \mathcal{S}_{K}(1) \rightarrow \mathbb{R}$ belongs to $\mathcal{E}$ (see [5]). It is natural to ask if this is true in the fusion frame setting for arbitrary $\mathbf{L}$. The answer is no (we will deal with this question in Remark 6.3), but one can assert something weaker. Let $\mathbf{W}$ be a local minimizer of $F F P_{\mathbf{w}}$. Theorem 4 in 4 says that there exists a generating family $\left\{\left\{f_{k, l}\right\}_{l=1}^{L_{k}}\right\}_{k=1}^{K}$ of $\left\{\pi_{W_{k}}\right\}_{k=1}^{K}$ (i.e. for each $k=1, \ldots, K,\left\{f_{k, l}\right\}_{l=1}^{L_{k}}$ is an orthonormal basis for $W_{k}$ ) that consists of eigenvectors of $S_{\mathbf{W}, \mathbf{w}}$, and that the elements of this family which are in $E_{j}$ are a $\lambda_{j}$-tight frame for $E_{j}$.

The next theorem establishes properties of the index sets $I_{j}$ along with properties of the families $\left\{W_{k}\right\}_{k \in I_{j}}, j=1, \ldots, J$ for pairs $(\mathbf{W}, \mathbf{w}) \in \mathcal{E}$ :

Theorem 3.1. Let $(\mathbf{W}, \mathbf{w}) \in \mathcal{E}$.

The following assertions hold:

(1) $\bigcup_{j=1}^{J} I_{j}=\{1, \ldots, K\}$.

(2) $k \in I_{j}$ if and only if $W_{k} \subseteq E_{j}$.

(3) If $j \neq j^{\prime}$, then $I_{j} \cap I_{j^{\prime}}=\emptyset$.

(4) $\lambda_{J}=\frac{1}{\operatorname{dim}\left(E_{J}\right)} \sum_{k \in I_{J}} w_{k}^{2} L_{k}$.

(5) For all $j=1, \ldots, J,\left\{W_{k}\right\}_{k \in I_{j}}$ is a $\lambda_{j}$-tight fusion frame for $E_{j}$.

(6) $I_{j}=\emptyset$ if and only if $\lambda_{j}=0$.

Proof. (1) It follows from the fact that $(\mathbf{W}, \mathbf{w}) \in \mathcal{E}$ and the definition of $I_{j}$.

(2) If $k \in I_{j}$ and $f \in W_{k}$, then $S_{\mathbf{W}, \mathbf{w}} f=S_{\mathbf{W}, \mathbf{w}} \pi_{W_{k}} f=\lambda_{j} \pi_{W_{k}} f=\lambda_{j} f$. Thus $f \in E_{j}$. Conversely, suppose that $W_{k} \subseteq E_{j}$. If $f \in \mathcal{H}, S_{\mathbf{W}, \mathbf{w}} \pi_{W_{k}} f=\lambda_{j} \pi_{W_{k}} f$. Thus $k \in I_{j}$.

(3) It follows from (2) and the orthogonality of the eigenspaces $E_{j}$. 
(4) Let $\left\{e_{l}\right\}_{l=1}^{\operatorname{dim}\left(E_{J}\right)}$ be an orthonormal basis for $E_{J}$ and $\left\{e_{l}\right\}_{l=\operatorname{dim}\left(E_{J}\right)+1}^{d}$ be an orthonormal basis for $E_{J}^{\perp}$. By part (2), $P_{k} e_{l}=0$ for $k \in I_{J}$ and $l \in\left\{\operatorname{dim}\left(E_{J}\right)+\right.$ $1, \ldots, d\}$, and $P_{k} e_{l}=0$ for $k \notin I_{J}$ and $l \in\left\{1, \ldots, \operatorname{dim}\left(E_{J}\right)\right\}$, hence

$$
\begin{aligned}
\sum_{k \in I_{J}} w_{k}^{2} L_{k} & =\sum_{k \in I_{J}} w_{k}^{2} \operatorname{tr}\left(P_{k}\right)=\sum_{k \in I_{J}} w_{k}^{2} \sum_{l=1}^{d}\left\langle P_{k} e_{l}, e_{l}\right\rangle=\sum_{k \in I_{J}} w_{k}^{2} \sum_{l=1}^{\operatorname{dim}\left(E_{J}\right)}\left\langle P_{k} e_{l}, e_{l}\right\rangle \\
& =\sum_{l=1}^{\operatorname{dim}\left(E_{J}\right)}\left\langle\sum_{k=1}^{K} w_{k}^{2} P_{k} e_{l}, e_{l}\right\rangle=\sum_{l=1}^{\operatorname{dim}\left(E_{J}\right)}\left\langle S_{\mathbf{W}, \mathbf{w}} e_{l}, e_{l}\right\rangle \\
& =\lambda_{J} \sum_{l=1}^{\operatorname{dim}\left(E_{J}\right)}\left\langle I_{\mathcal{H}} e_{l}, e_{l}\right\rangle=\lambda_{J} \sum_{l=1}^{\operatorname{dim}\left(E_{J}\right)}\left\|e_{l}\right\|^{2}=\lambda_{J} \operatorname{dim}\left(E_{J}\right) .
\end{aligned}
$$

(5) Let $j \in\{1, \ldots, J\}$ and $f \in E_{j}$. By (1) and (2), if $k \in\{1, \ldots, K\} \cap I_{j}^{c}$ then $W_{k} \subseteq E_{j^{\prime}}$ for some $j^{\prime} \neq j$ and consequently $\pi_{W_{k}} f=0$. So,

$$
\lambda_{j} f=S_{\mathbf{W}, \mathbf{w}} f=\sum_{k=1}^{K} w_{k}^{2} \pi_{W_{k}} f=\sum_{k \in I_{j}} w_{k}^{2} \pi_{W_{k}} f .
$$

This shows that $\left\{W_{k}\right\}_{k \in I_{j}}$ is a $\lambda_{j}$-tight fusion frame for $E_{j}$.

(6) We have $I_{j_{0}}=\emptyset$ if and only of $\forall k \in\{1, \ldots, K\}, W_{k} \subseteq E_{j_{0}}^{\perp}$, or equivalently, $E_{j_{0}} \subseteq R\left(T_{\mathbf{W}, \mathbf{w}}\right)^{\perp}=N\left(S_{\mathbf{W}, \mathbf{w}}\right)$ i. e. $\lambda_{j_{0}}=0$

\section{Minimizers in $\mathcal{E}$}

In the vectorial case every sequence, which is a local minimizer of the frame potential, consists of elements that are eigenvectors of the frame operator. Motivated by that, in this section we describe the structure of the local minimizers $\mathbf{W}$ of the fusion frame potential with fixed dimensions and weights such that $(\mathbf{W}, \mathbf{w}) \in \mathcal{E}$. In this case we can give a complete description as it was done in [1, 5, for the frame potential. Moreover, as in this works, we relate these results to the existence of tight fusion frames in terms of a fundamental inequality that must be satisfied by the weights and the dimension of the subspaces.

To prove Theorem 4.2 we use the following auxiliary Lemma.

Lemma 4.1. Let $\left\{f_{l}\right\}_{l=1}^{L} \subset \mathbb{F}^{d}$ be an orthonormal set. Let $\left\{z_{l}\right\}_{l=1}^{L} \subset \mathbb{C}$ be a nonzero sequence such that $\left|z_{l}\right| \leq \frac{1}{2}$ for each $l=1, \ldots, L$. Let $h \in \mathbb{F}^{d}$ such that $\|h\|=1$ and $\left\langle h, f_{l}\right\rangle=0$ for each $l=1, \ldots, L$. For each $t \in(-1,1)$, let the matrices $A(t), F, H, \widetilde{F}$ and $D$ given by

for $l=1, \ldots, L$, and

$$
\begin{gathered}
A(t)(:, l)=\left(1-t^{2}\left|z_{l}\right|^{2}\right)^{\frac{1}{2}} f_{l}+t z_{l} h, \\
F(:, l)=f_{l}, \\
H(:, l)=z_{l} h, \\
\widetilde{F}(:, l)=-\left|z_{l}\right|^{2} f_{l},
\end{gathered}
$$

Then

$$
D\left(l, l^{\prime}\right)=-2 \delta_{l, l^{\prime}} z_{l} \overline{z_{l^{\prime}}} \text { for } l, l^{\prime}=1, \ldots, L .
$$

(1) $\operatorname{dim}(R(A(t)))=L$.

(2) $\left.\frac{d}{d t} \pi_{R(A(t))}\right|_{t=0}=H F^{*}+F H^{*}$.

(3) $\left.\frac{d^{2}}{d t^{2}} \pi_{R(A(t))}\right|_{t=0}=\widetilde{F} F^{*}+2 H H^{*}+F D F^{*}+F \widetilde{F}^{*}$. 
Proof. To prove (1) we are going to see that the columns of $A(t)$ are linearly independent. To see this, let $\left\{c_{l}\right\}_{l=1}^{L} \subset \mathbb{C}$ be such that $\sum_{l=1}^{L} c_{l} A(t)(:, l)=0$, or equivalently, $\sum_{l=1}^{L} c_{l}\left(1-t^{2}\left|z_{l}\right|^{2}\right)^{\frac{1}{2}} f_{l}+t \sum_{l=1}^{L} c_{l} z_{l} h=0$. Let $l_{0} \in\{1, \ldots, L\}$. Taking into account that $\left\langle f_{l_{0}}, f_{l}\right\rangle=\delta_{l, l_{0}}$ for all $l \in\{1, \ldots, L\}$ and $\left\langle f_{l_{0}}, h\right\rangle=0$, then $0=\left\langle f_{l_{0}}, \sum_{l=1}^{L} c_{l}\left(1-t^{2}\left|z_{l}\right|^{2}\right)^{\frac{1}{2}} f_{l}+t \sum_{l=1}^{L} c_{l} z_{l} h\right\rangle=c_{l_{0}}\left(1-t^{2}\left|z_{l}\right|^{2}\right)^{\frac{1}{2}}$. Since $t \in(-1,1)$ and $\left|z_{l}\right| \leq \frac{1}{2}$ for each $k \in\{1, \ldots, K\}$ and $l=1, \ldots, L$, it follows that $c_{l_{0}}=0$. Now we are going to prove (2) and (3). We begin noting that

$$
\begin{aligned}
& \left.\frac{d}{d t} A(t)\right|_{t=0}=H \\
& \left.\frac{d^{2}}{d t^{2}} A(t)\right|_{t=0}=\widetilde{F}
\end{aligned}
$$

and

$$
\left[A^{*}(t) A(t)\right]\left(l, l^{\prime}\right)=\delta_{l, l^{\prime}}+\left(1-\delta_{l, l^{\prime}}\right) t^{2} z_{l} \overline{z_{l^{\prime}}} \text { for } l, l^{\prime}=1, \ldots, L .
$$

Let $\mathbb{L}:=\{1, \ldots, L\}, B \subset \mathbb{L}, \mathbb{L}^{s} \backslash B=\left\{\left(l_{1}, \ldots, l_{s}\right) \in \mathbb{L} \backslash B \times \ldots \times \mathbb{L} \backslash B: l_{1}<\ldots<l_{s}\right\}$. We write $\mathbb{L}^{s} \backslash \emptyset:=\mathbb{L}^{s}$. It is easy to check that the entry $(i, i)$ of $\left[A^{*}(t) A(t)\right]^{-1}$ is

$$
\frac{1+\sum_{s=2}^{L-1}(-1)^{s-1}(s-1) t^{2 s} \sum_{\left(l_{1}, \ldots, l_{s}\right) \in \mathbb{L}^{s} \backslash\{i\}}\left|z_{l_{1}}\right|^{2} \ldots\left|z_{l_{s}}\right|^{2}}{1+\sum_{s=2}^{L}(-1)^{s-1}(s-1) t^{2 s} \sum_{\left(l_{1}, \ldots, l_{s}\right) \in \mathbb{L}^{s}}\left|z_{l_{1}}\right|^{2} \ldots\left|z_{l_{s}}\right|^{2}}
$$

and the entry $(i, j)$ with $i \neq j$ is

Therefore,

$$
\frac{-t^{2} \bar{z}_{i} z_{j}\left(1+\sum_{s=1}^{L-2}(-1)^{s} t^{2 s} \sum_{\left(l_{1}, \ldots, l_{s}\right) \in \mathbb{L}^{s} \backslash\{i, j\}}\left|z_{l_{1}}\right|^{2} \ldots\left|z_{l_{s}}\right|^{2}\right)}{1+\sum_{s=2}^{L}(-1)^{s-1}(s-1) t^{2 s} \sum_{\left(l_{1}, \ldots, l_{s}\right) \in \mathbb{L}^{s}}\left|z_{l_{1}}\right|^{2} \ldots\left|z_{l_{s}}\right|^{2}} .
$$

$$
\left.\frac{d}{d t}\left(\left[A^{*}(t) A(t)\right]^{-1}\right)\right|_{t=0}=0
$$

and

$$
\left.\frac{d^{2}}{d t^{2}}\left(\left[A^{*}(t) A(t)\right]^{-1}\right)\right|_{t=0}=D .
$$

We have $\pi_{\mathrm{R}(A(t))}=A(t)\left[A^{*}(t) A(t)\right]^{-1} A^{*}(t)$. Thus, from (4.1), (4.2), (4.3) and (4.4) we obtain (2) and (3).

Theorem 4.2. If $\mathbf{W}$ is a local minimizer of $F F P_{\mathbf{w}}$ such that $(\mathbf{W}, \mathbf{w}) \in \mathcal{E}$, then $\left\{W_{k}\right\}_{k \in I_{j}}$ is a direct sum for all $j<J$.

Proof. Suppose that there exists $1 \leq j \leq J-1$ such that $\left\{W_{k}\right\}_{k \in I_{j}}$ is not a direct sum. Then there exists a linearly dependent set $\left\{\left\{f_{k, l}\right\}_{l=1}^{L_{k}}\right\}_{k \in I_{j}}$ such that $\left\{f_{k, l}\right\}_{l=1}^{L_{k}}$ 
is an orthonormal basis for $W_{k}, k \in I_{j}$. Consequently, there exists a nonzero sequence $\left\{\left\{z_{k, l}\right\}_{k=1}^{L_{k}}\right\}_{k \in I_{j}} \subset \mathbb{C}$ with $\left|z_{k, l}\right| \leq \frac{1}{2}$ for all $k \in I_{j}, k=1, \ldots, L_{k}$ such that

$$
\sum_{k \in I_{j}} w_{k}^{2} \sum_{l=1}^{L_{k}} \bar{z}_{k, l} f_{k, l}=0 .
$$

The eigenspace $E_{J}$ of $S_{\mathbf{W}, \mathbf{w}}$ is at least 1-dimensional. So, there exists $h \in E_{J}$ with $\|h\|=1$. Using $\left\{f_{k, l}\right\}_{l=1}^{L_{k}},\left\{z_{k, l}\right\}_{k=1}^{L_{k}}$ and $h$, we define $A_{k}(t), F_{k}, H_{k}, \widetilde{F}_{k}$ and $D_{k}$ as in Lemma 4.1 where we now consider $k \in I_{j}$ instead of $k \in\{1, \ldots, K\}$.

Since the columns of $F_{k}$ are orthonormal,

$$
F_{k}^{*} F_{k}=I \text { for } k \in I_{j} .
$$

By Theorem $3.1(2)$, if $k \in I_{j}$ then $\left\{\left\{f_{k, l}\right\}_{l=1}^{L_{k}}\right\}_{k \in I_{j}} \subset E_{j}$. Thus, the conclusions of Lemma 4.1 hold and

$$
H_{k}^{*} F_{k^{\prime}}=0 \text { for } k, k^{\prime} \in I_{j}
$$

Let

$$
V_{k}(t)= \begin{cases}\mathrm{R}\left(A_{k}(t)\right), & \text { si } k \in I_{j} \\ W_{k}, & \text { si } k \notin I_{j}\end{cases}
$$

The sequence of continuous subspaces curves $\left\{V_{k}(t)\right\}_{k=1}^{K}$ satisfies that $\left\{V_{k}(0)\right\}_{k=1}^{K}=$ W and, by Lemma 4.1(1), $\left\{V_{k}(t)\right\}_{k=1}^{K} \in \mathcal{S}_{K}(\mathbf{L})$ for all $t \in(-1,1)$. We are going to see that

$$
\left.\frac{d}{d t} F F P_{\mathbf{w}}\left(\left\{V_{k}(t)\right\}_{k=1}^{K}\right)\right|_{t=0}=0 \text { and }\left.\frac{d^{2}}{d t^{2}} F F P_{\mathbf{w}}\left(\left\{V_{k}(t)\right\}_{k=1}^{K}\right)\right|_{t=0}<0 .
$$

Therefore,

$$
F F P_{\mathbf{w}}\left(\left\{V_{k}(t)\right\}_{k=1}^{K}\right)<F F P_{\mathbf{w}}(\mathbf{W})
$$

for all $t$ in a neighborhood of 0 , contradicting the hypothesis that $\mathbf{W}$ is a local minimum of $F F P_{\mathbf{w}}$.

To simplify the exposition in the rest of the proof we set $P_{k}:=\pi_{W_{k}}=F_{k} F_{k}^{*}$ and $\tilde{P}_{k}(t):=\pi_{V_{k}(t)}$.

We have

$$
\begin{aligned}
& \frac{d}{d t} F_{F} P_{\mathbf{w}}\left(\left\{V_{k}(t)\right\}_{k=1}^{K}\right)=\frac{d}{d t} \sum_{k=1}^{K} \sum_{k^{\prime}=1}^{K} w_{k}^{2} w_{k^{\prime}}^{2} \operatorname{tr}\left(\left[\widetilde{P}_{k}(t) \widetilde{P}_{k^{\prime}}(t)\right]\left[\widetilde{P}_{k^{\prime}}(t) \widetilde{P}_{k}(t)\right]\right)= \\
& =\sum_{k=1}^{K} \sum_{k^{\prime}=1}^{K} w_{k}^{2} w_{k^{\prime}}^{2} \operatorname{tr}\left(\widetilde{P}_{k}^{\prime}(t) \widetilde{P}_{k^{\prime}}(t) \widetilde{P}_{k^{\prime}}(t) \widetilde{P}_{k}(t)\right)+\sum_{k=1}^{K} \sum_{k^{\prime}=1}^{K} w_{k}^{2} w_{k^{\prime}}^{2} \operatorname{tr}\left(\widetilde{P}_{k}(t) \widetilde{P}_{k^{\prime}}^{\prime}(t) \widetilde{P}_{k^{\prime}}(t) \widetilde{P}_{k}(t)\right) \\
& \quad+\sum_{k=1}^{K} \sum_{k^{\prime}=1}^{K} w_{k}^{2} w_{k^{\prime}}^{2} \operatorname{tr}\left(\widetilde{P}_{k}(t) \widetilde{P}_{k^{\prime}}(t) \widetilde{P}_{k^{\prime}}^{\prime}(t) \widetilde{P}_{k}(t)\right)+\sum_{k=1}^{K} \sum_{k^{\prime}=1}^{K} w_{k}^{2} w_{k^{\prime}}^{2} \operatorname{tr}\left(\widetilde{P}_{k}(t) \widetilde{P}_{k^{\prime}}(t) \widetilde{P}_{k^{\prime}}(t) \widetilde{P}_{k}^{\prime}(t)\right) \\
& =2\left(\sum_{k=1}^{K} \sum_{k^{\prime}=1}^{K} w_{k}^{2} w_{k^{\prime}}^{2} \operatorname{tr}\left(\widetilde{P}_{k}^{\prime}(t) \widetilde{P}_{k^{\prime}}(t) \widetilde{P}_{k}(t)\right)+\sum_{k=1}^{K} \sum_{k^{\prime}=1}^{K} w_{k}^{2} w_{k^{\prime}}^{2} \operatorname{tr}\left(\widetilde{P}_{k}^{\prime}(t) \widetilde{P}_{k}(t) \widetilde{P}_{k^{\prime}}(t)\right)\right)
\end{aligned}
$$


Then

$$
\begin{aligned}
\left.\frac{d}{d t} F F P_{\mathbf{w}}\left(\left\{V_{k}(t)\right\}_{k=1}^{K}\right)\right|_{t=0} & =2\left(\sum_{k \in I_{j}} \sum_{k^{\prime}=1}^{K} w_{k}^{2} w_{k^{\prime}}^{2} \operatorname{tr}\left(\widetilde{P}_{k}^{\prime}(0) P_{k^{\prime}} P_{k}\right)+\sum_{k \in I_{j}} \sum_{k^{\prime}=1}^{K} w_{k}^{2} w_{k^{\prime}}^{2} \operatorname{tr}\left(\widetilde{P}_{k}^{\prime}(0) P_{k} P_{k^{\prime}}\right)\right) \\
& =2\left(\sum_{k \in I_{j}} w_{k}^{2} \operatorname{tr}\left(\widetilde{P}_{k}^{\prime}(0) S_{\mathbf{W}, \mathbf{w}} P_{k}\right)+\sum_{k \in I_{j}} w_{k}^{2} \operatorname{tr}\left(\widetilde{P}_{k}^{\prime}(0) P_{k} S_{\mathbf{W}, \mathbf{w}}\right)\right)
\end{aligned}
$$

Using that $(\mathbf{W}, \mathbf{w}) \in \mathcal{E}$, Lemma 4.1 (2), 4.6) and 4.7),

$$
\begin{aligned}
\left.\frac{d}{d t}\left(F F P_{\mathbf{w}}\left(\left\{\tilde{P}_{k}\right\}_{k=1}^{K}\right)\right)\right|_{t=0} & =2\left(\lambda_{j} \sum_{k \in I_{j}} w_{k}^{2} \operatorname{tr}\left(\widetilde{P}_{k}^{\prime}(0) P_{k}\right)+\lambda_{j} \sum_{k \in I_{j}} w_{k}^{2} \operatorname{tr}\left(\widetilde{P}_{k}^{\prime}(0) P_{k}\right)\right) \\
& =4 \lambda_{j} \sum_{k \in I_{j}} w_{k}^{2} \operatorname{tr}\left(\left(H_{k} F_{k}^{*}+F_{k} H_{k}^{*}\right) F_{k} F_{k}^{*}\right) \\
& =4 \lambda_{j} \sum_{k \in I_{j}} w_{k}^{2} \operatorname{tr}\left(H_{k} F_{k}^{*}\right)=4 \lambda_{j} \sum_{k \in I_{j}} w_{k}^{2} \operatorname{tr}\left(F_{k}^{*} H_{k}\right)=0 .
\end{aligned}
$$

For the second derivative we have

$$
\begin{aligned}
\frac{d^{2}}{d t^{2}} F F P_{\mathbf{w}}\left(\left\{V_{k}(t)\right\}_{k=1}^{K}\right) & =\sum_{k=1}^{K} \sum_{k^{\prime}=1}^{K} w_{k}^{2} w_{k^{\prime}}^{2} \frac{d^{2}}{d t^{2}} \operatorname{tr}\left(\left[\widetilde{P}_{k}(t) \widetilde{P}_{k^{\prime}}(t)\right]\left[\widetilde{P}_{k^{\prime}}(t) \widetilde{P}_{k}(t)\right]\right) \\
& =2[a(t)+b(t)]
\end{aligned}
$$

where

$$
\begin{aligned}
a(t):= & \sum_{k=1}^{K} \sum_{k^{\prime}=1}^{K} w_{k}^{2} w_{k^{\prime}}^{2} \operatorname{tr}\left(\widetilde{P}_{k}^{\prime \prime}(t) \widetilde{P}_{k^{\prime}}(t) \widetilde{P}_{k}(t)\right)+\sum_{k=1}^{K} \sum_{k^{\prime}=1}^{K} w_{k}^{2} w_{k^{\prime}}^{2} \operatorname{tr}\left(\widetilde{P}_{k}^{\prime}(t) \widetilde{P}_{k^{\prime}}^{\prime}(t) \widetilde{P}_{k^{\prime}}(t) \widetilde{P}_{k}(t)\right)+ \\
& +\sum_{k=1}^{K} \sum_{k^{\prime}=1}^{K} w_{k}^{2} w_{k^{\prime}}^{2} \operatorname{tr}\left(\widetilde{P}_{k}^{\prime}(t) \widetilde{P}_{k^{\prime}}(t) \widetilde{P}_{k^{\prime}}^{\prime}(t) \widetilde{P}_{k}(t)\right)+\sum_{k=1}^{K} \sum_{k^{\prime}=1}^{K} w_{k}^{2} w_{k^{\prime}}^{2} \operatorname{tr}\left(\widetilde{P}_{k}^{\prime}(t) \widetilde{P}_{k^{\prime}}(t) \widetilde{P}_{k}^{\prime}(t)\right)
\end{aligned}
$$

and

$$
\begin{aligned}
b(t):= & \sum_{k=1}^{K} \sum_{k^{\prime}=1}^{K} w_{k}^{2} w_{k^{\prime}}^{2} \operatorname{tr}\left(\widetilde{P}_{k}^{\prime}(t) \widetilde{P}_{k^{\prime}}^{\prime}(t) \widetilde{P}_{k^{\prime}}(t) \widetilde{P}_{k}(t)\right)+\sum_{k=1}^{K} \sum_{k^{\prime}=1}^{K} w_{k}^{2} w_{k^{\prime}}^{2} \operatorname{tr}\left(\widetilde{P}_{k}(t) \widetilde{P}_{k^{\prime}}^{\prime \prime}(t) \widetilde{P}_{k^{\prime}}(t)\right)+ \\
& +\sum_{k=1}^{K} \sum_{k^{\prime}=1}^{K} w_{k}^{2} w_{k^{\prime}}^{2} \operatorname{tr}\left(\widetilde{P}_{k}(t) \widetilde{P}_{k^{\prime}}^{\prime}(t) \widetilde{P}_{k^{\prime}}^{\prime}(t)\right)+\sum_{k=1}^{K} \sum_{k^{\prime}=1}^{K} w_{k}^{2} w_{k^{\prime}}^{2} \operatorname{tr}\left(\widetilde{P}_{k}(t) \widetilde{P}_{k^{\prime}}^{\prime}(t) \widetilde{P}_{k^{\prime}}(t) \widetilde{P}_{k}^{\prime}(t)\right) .
\end{aligned}
$$


Using that $(\mathbf{W}, \mathbf{w}) \in \mathcal{E}$, we obtain

$$
\begin{aligned}
a(0)= & \sum_{k \in I_{j}} \sum_{k^{\prime}=1}^{K} w_{k}^{2} w_{k^{\prime}}^{2} \operatorname{tr}\left(\widetilde{P}_{k}^{\prime \prime}(0) \widetilde{P}_{k^{\prime}} \widetilde{P}_{k}\right)+\sum_{k \in I_{j}} \sum_{k^{\prime} \in I_{j}} w_{k}^{2} w_{k^{\prime}}^{2} \operatorname{tr}\left(\widetilde{P}_{k}^{\prime}(0) \widetilde{P}_{k^{\prime}}^{\prime}(0) \widetilde{P}_{k^{\prime}} \widetilde{P}_{k}\right)+ \\
& +\sum_{k \in I_{j}} \sum_{k^{\prime} \in I_{j}} w_{k}^{2} w_{k^{\prime}}^{2} \operatorname{tr}\left(\widetilde{P}_{k}^{\prime}(0) \widetilde{P}_{k^{\prime}} \widetilde{P}_{k^{\prime}}^{\prime}(0) \widetilde{P}_{k}\right)+\sum_{k \in I_{j}} \sum_{k^{\prime}=1}^{K} w_{k}^{2} w_{k^{\prime}}^{2} \operatorname{tr}\left(\widetilde{P}_{k}^{\prime}(0) \widetilde{P}_{k^{\prime}} \widetilde{P}_{k}^{\prime}(0)\right) \\
= & \sum_{k \in I_{j}} w_{k}^{2} \operatorname{tr}\left(\widetilde{P}_{k}^{\prime \prime}(0) S \mathbf{W}, \mathbf{w} \widetilde{P}_{k}\right)+\sum_{k \in I_{j}} \sum_{k^{\prime} \in I_{j}} w_{k}^{2} w_{k^{\prime}}^{2} \operatorname{tr}\left(\widetilde{P}_{k}^{\prime}(0) \widetilde{P}_{k^{\prime}}^{\prime}(0) \widetilde{P}_{k^{\prime}} \widetilde{P}_{k}\right)+ \\
& +\sum_{k \in I_{j}} \sum_{k^{\prime} \in I_{j}} w_{k}^{2} w_{k^{\prime}}^{2} \operatorname{tr}\left(\widetilde{P}_{k}^{\prime}(0) \widetilde{P}_{k^{\prime}} \widetilde{P}_{k^{\prime}}^{\prime}(0) \widetilde{P}_{k}\right)+\sum_{k \in I_{j}} w_{k}^{2} \operatorname{tr}\left(\widetilde{P}_{k}^{\prime}(0) S_{\mathbf{W}, \mathbf{w}} \widetilde{P}_{k}^{\prime}(0)\right) \\
= & \lambda_{j} \sum_{k \in I_{j}} w_{k}^{2} \operatorname{tr}\left(\widetilde{P}_{k}^{\prime \prime}(0) \widetilde{P}_{k}\right)+\sum_{k \in I_{j}} \sum_{k^{\prime} \in I_{j}} w_{k}^{2} w_{k^{\prime}}^{2} \operatorname{tr}\left(\widetilde{P}_{k}^{\prime}(0) \widetilde{P}_{k^{\prime}}^{\prime}(0) \widetilde{P}_{k^{\prime}} \widetilde{P}_{k}\right) \\
(4.9) \quad & +\sum_{k \in I_{j}} \sum_{k^{\prime} \in I_{j}} w_{k}^{2} w_{k^{\prime}}^{2} \operatorname{tr}\left(\widetilde{P}_{k}^{\prime}(0) \widetilde{P}_{k^{\prime}} \widetilde{P}_{k^{\prime}}^{\prime}(0) \widetilde{P}_{k}\right)+\sum_{k \in I_{j}} w_{k}^{2} \operatorname{tr}\left(\widetilde{P}_{k}^{\prime}(0) S_{\mathbf{W}, \mathbf{w}} \widetilde{P}_{k}^{\prime}(0)\right)
\end{aligned}
$$

and

$$
\begin{aligned}
b(0)= & \sum_{k \in I_{j}} \sum_{k^{\prime} \in I_{j}} w_{k}^{2} w_{k^{\prime}}^{2} \operatorname{tr}\left(\widetilde{P}_{k}^{\prime}(0) \widetilde{P}_{k^{\prime}}^{\prime}(0) \widetilde{P}_{k^{\prime}} \widetilde{P}_{k}\right)+\sum_{k=1}^{K} \sum_{k^{\prime} \in I_{j}} w_{k}^{2} w_{k^{\prime}}^{2} \operatorname{tr}\left(\widetilde{P}_{k} \widetilde{P}_{k^{\prime}}^{\prime \prime}(0) \widetilde{P}_{k^{\prime}}\right)+ \\
& +\sum_{k=1}^{K} \sum_{k^{\prime} \in I_{j}} w_{k}^{2} w_{k^{\prime}}^{2} \operatorname{tr}\left(\widetilde{P}_{k} \widetilde{P}_{k^{\prime}}^{\prime}(0) \widetilde{P}_{k^{\prime}}^{\prime}(0)\right)+\sum_{k \in I_{j}} \sum_{k^{\prime} \in I_{j}} w_{k}^{2} w_{k^{\prime}}^{2} \operatorname{tr}\left(\widetilde{P}_{k} \widetilde{P}_{k^{\prime}}^{\prime}(0) \widetilde{P}_{k^{\prime}} \widetilde{P}_{k}^{\prime}(0)\right) \\
= & \sum_{k \in I_{j}} \sum_{k^{\prime} \in I_{j}} w_{k}^{2} w_{k^{\prime}}^{2} \operatorname{tr}\left(\widetilde{P}_{k}^{\prime}(0) \widetilde{P}_{k^{\prime}}^{\prime}(0) \widetilde{P}_{k^{\prime}} \widetilde{P}_{k}\right)+\sum_{k^{\prime} \in I_{j}} w_{k^{\prime}}^{2} \operatorname{tr}\left(S \mathbf{W}, \mathbf{w} \widetilde{P}_{k^{\prime}}^{\prime \prime}(0) \widetilde{P}_{k^{\prime}}\right)+ \\
& +\sum_{k^{\prime} \in I_{j}} w_{k^{\prime}}^{2} \operatorname{tr}\left(S_{\mathbf{W}, \mathbf{w}} \widetilde{P}_{k^{\prime}}^{\prime}(0) \widetilde{P}_{k^{\prime}}^{\prime}(0)\right)+\sum_{k \in I_{j}} \sum_{k^{\prime} \in I_{j}} w_{k}^{2} w_{k^{\prime}}^{2} \operatorname{tr}\left(\widetilde{P}_{k} \widetilde{P}_{k^{\prime}}^{\prime}(0) \widetilde{P}_{k^{\prime}} \widetilde{P}_{k}^{\prime}(0)\right) \\
= & \lambda_{j} \sum_{k \in I_{j}} w_{k}^{2} \operatorname{tr}\left(\widetilde{P}_{k}^{\prime \prime}(0) \widetilde{P}_{k}\right)+\sum_{k \in I_{j}} \sum_{k^{\prime} \in I_{j}} w_{k}^{2} w_{k^{\prime}}^{2} \operatorname{tr}\left(\widetilde{P}_{k}^{\prime}(0) \widetilde{P}_{k^{\prime}}^{\prime}(0) \widetilde{P}_{k^{\prime}} \widetilde{P}_{k}\right) \\
(4.10) & +\sum_{k \in I_{j}} w_{k}^{2} \operatorname{tr}\left(\widetilde{P}_{k}^{\prime}(0) S S_{\mathbf{W}, \mathbf{w}} \widetilde{P}_{k}^{\prime}(0)\right)+\sum_{k \in I_{j}} \sum_{k^{\prime} \in I_{j}} w_{k}^{2} w_{k^{\prime}}^{2} \operatorname{tr}\left(\widetilde{P}_{k} \widetilde{P}_{k^{\prime}}^{\prime}(0) \widetilde{P}_{k^{\prime}} \widetilde{P}_{k}^{\prime}(0)\right) .
\end{aligned}
$$

Using (4.6) and (4.7) along with Lemma 4.1(2)-(3), that $\operatorname{tr}\left(D_{k}\right)=0$ and (4.5), we can rewrite each sum in (4.9) and (4.10) obtaining

$$
\begin{aligned}
\lambda_{j} \sum_{k \in I_{j}} w_{k}^{2} \operatorname{tr}\left(\widetilde{P}_{k}^{\prime \prime}(0) \widetilde{P}_{k}\right) & =\lambda_{j} \sum_{k \in I_{j}} w_{k}^{2} \operatorname{tr}\left(\left[\widetilde{F}_{k} F_{k}^{*}+2 H_{k} H_{k}^{*}+F_{k} D_{k} F_{k}^{*}+F_{k} \widetilde{F}_{k}^{*}\right] F_{k} F_{k}^{*}\right) \\
& =\lambda_{j} \sum_{k \in I_{j}} w_{k}^{2} \operatorname{tr}\left(\widetilde{F}_{k} F_{k}^{*}+D_{k}+\widetilde{F}_{k}^{*} F_{k}\right) \\
& =2 \lambda_{j} \sum_{k \in I_{j}} w_{k}^{2} \operatorname{tr}\left(\widetilde{F}_{k} F_{k}^{*}\right)=-2 \lambda_{j} \sum_{k \in I_{j}} w_{k}^{2} \sum_{l=1}^{L_{k}}\left|z_{k, l}\right|^{2},
\end{aligned}
$$




$$
\begin{aligned}
& \sum_{k \in I_{j}} \sum_{k^{\prime} \in I_{j}} w_{k}^{2} w_{k^{\prime}}^{2} \operatorname{tr}\left(\widetilde{P}_{k}^{\prime}(0) \widetilde{P}_{k^{\prime}}^{\prime}(0) \widetilde{P}_{k^{\prime}} \widetilde{P}_{k}\right)= \\
& =\sum_{k \in I_{j}} \sum_{k^{\prime} \in I_{j}} w_{k}^{2} w_{k^{\prime}}^{2} \operatorname{tr}\left(\left(H_{k} F_{k}^{*}+F_{k} H_{k}^{*}\right)\left(H_{k^{\prime}} F_{k^{\prime}}^{*}+F_{k^{\prime}} H_{k^{\prime}}^{*}\right) F_{k^{\prime}} F_{k^{\prime}}^{*} F_{k} F_{k}^{*}\right) \\
& =\sum_{k \in I_{j}} \sum_{k^{\prime} \in I_{j}} w_{k}^{2} w_{k^{\prime}}^{2} \operatorname{tr}\left(F_{k} F_{k}^{*}\left(H_{k} F_{k}^{*}+F_{k} H_{k}^{*}\right)\left(H_{k^{\prime}} F_{k^{\prime}}^{*}+F_{k^{\prime}} H_{k^{\prime}}^{*}\right) F_{k^{\prime}} F_{k^{\prime}}^{*}\right) \\
& =\sum_{k \in I_{j}} \sum_{k^{\prime} \in I_{j}} w_{k}^{2} w_{k^{\prime}}^{2} \operatorname{tr}\left(F_{k} H_{k}^{*} H_{k^{\prime}} F_{k^{\prime}}^{*}\right)=\left\|\sum_{k \in I_{j}} w_{k}^{2} \sum_{l=1}^{L_{k}} \overline{z_{k, l}} f_{k, l}\right\|^{2}=0, \\
& \sum_{k \in I_{j}} \sum_{k^{\prime} \in I_{j}} w_{k}^{2} w_{k^{\prime}}^{2} \operatorname{tr}\left(\widetilde{P}_{k}^{\prime}(0) \widetilde{P}_{k^{\prime}} \widetilde{P}_{k^{\prime}}^{\prime}(0) \widetilde{P}_{k}\right)= \\
& =\sum_{k \in I_{j}} \sum_{k^{\prime} \in I_{j}} w_{k}^{2} w_{k^{\prime}}^{2} \operatorname{tr}\left(\widetilde{P}_{k} \widetilde{P}_{k}^{\prime}(0) \widetilde{P}_{k^{\prime}} \widetilde{P}_{k^{\prime}}^{\prime}(0)\right) \\
& =\sum_{k \in I_{j}} \sum_{k^{\prime} \in I_{j}} w_{k}^{2} w_{k^{\prime}}^{2} \operatorname{tr}\left(F_{k} F_{k}^{*}\left(H_{k} F_{k}^{*}+F_{k} H_{k}^{*}\right) F_{k^{\prime}} F_{k^{\prime}}^{*}\left(H_{k^{\prime}} F_{k^{\prime}}^{*}+F_{k^{\prime}} H_{k^{\prime}}^{*}\right)\right) \\
& =\sum_{k \in I_{j}} \sum_{k^{\prime} \in I_{j}} w_{k}^{2} w_{k^{\prime}}^{2} \operatorname{tr}\left(F_{k} H_{k}^{*} F_{k^{\prime}} H_{k^{\prime}}^{*}\right)=0, \\
& \sum_{k \in I_{j}} w_{k}^{2} \operatorname{tr}\left(\widetilde{P}_{k}^{\prime}(0) S_{\mathbf{W}, \mathbf{w}} \widetilde{P}_{k}^{\prime}(0)\right)=\sum_{k \in I_{j}} w_{k}^{2} \operatorname{tr}\left(\left(H_{k} F_{k}^{*}+F_{k} H_{k}^{*}\right) S_{\mathbf{W}, \mathbf{w}}\left(H_{k} F_{k}^{*}+F_{k} H_{k}^{*}\right)\right) \\
& =\sum_{k \in I_{j}} w_{k}^{2} \operatorname{tr}\left(\left(H_{k} F_{k}^{*}+F_{k} H_{k}^{*}\right)\left(\lambda_{J} H_{k} F_{k}^{*}+\lambda_{j} F_{k} H_{k}^{*}\right)\right) \\
& =\sum_{k \in I_{j}} w_{k}^{2}\left(\lambda_{j} \operatorname{tr}\left(H_{k} H_{k}^{*}\right)+\lambda_{J} \operatorname{tr}\left(H_{k}^{*} H_{k}\right)\right) \\
& =\left(\lambda_{j}+\lambda_{J}\right) \sum_{k \in I_{j}} w_{k}^{2} \operatorname{tr}\left(H_{k}^{*} H_{k}\right)=\left(\lambda_{j}+\lambda_{J}\right) \sum_{k \in I_{j}} w_{k}^{2} \sum_{l=1}^{L_{k}}\left|z_{k, l}\right|^{2},
\end{aligned}
$$

and

$\sum_{k \in I_{j}} \sum_{k^{\prime} \in I_{j}} w_{k}^{2} w_{k^{\prime}}^{2} \operatorname{tr}\left(\widetilde{P}_{k} \widetilde{P}_{k^{\prime}}^{\prime}(0) \widetilde{P}_{k^{\prime}} \widetilde{P}_{k}^{\prime}(0)\right)=$

$$
\begin{aligned}
& =\sum_{k \in I_{j}} \sum_{k^{\prime} \in I_{j}} w_{k}^{2} w_{k^{\prime}}^{2} \operatorname{tr}\left(F_{k} F_{k}^{*}\left(H_{k^{\prime}} F_{k^{\prime}}^{*}+F_{k^{\prime}} H_{k^{\prime}}^{*}\right) F_{k^{\prime}} F_{k^{\prime}}^{*}\left(H_{k} F_{k}^{*}+F_{k} H_{k}^{*}\right)\right) \\
& =\sum_{k \in I_{j}} \sum_{k^{\prime} \in I_{j}} w_{k}^{2} w_{k^{\prime}}^{2} \operatorname{tr}\left(\left(F_{k} F_{k}^{*} F_{k^{\prime}} H_{k^{\prime}}^{*}\right)\left(F_{k^{\prime}} F_{k^{\prime}}^{*} F_{k} H_{k}^{*}\right)\right)=0 .
\end{aligned}
$$

By (4.11), (4.12), (4.13) and (4.14) in (4.9), and (4.11), (4.12), (4.14) and (4.15) in (4.10),

$$
\begin{aligned}
a(0)=b(0) & =-2 \lambda_{j} \sum_{k \in I_{j}} w_{k}^{2} \sum_{l=1}^{L_{k}}\left|z_{k, l}\right|^{2}+\left(\lambda_{j}+\lambda_{J}\right) \sum_{k \in I_{j}} w_{k}^{2} \sum_{l=1}^{L_{k}}\left|z_{k, l}\right|^{2} \\
& =\left(\lambda_{J}-\lambda_{j}\right) \sum_{k \in I_{j}} w_{k}^{2} \sum_{l=1}^{L_{k}}\left|z_{k, l}\right|^{2}<0,
\end{aligned}
$$


and consequently $\left.\frac{d^{2}}{d t^{2}} F F P_{\mathbf{w}}\left(\left\{V_{k}(t)\right\}_{k=1}^{K}\right)\right|_{t=0}=2[a(0)+b(0)]<0$.

Theorem 4.3. If $\mathbf{W}$ is a local minimizer of $F F P_{\mathbf{w}}$ such that $(\mathbf{W}, \mathbf{w}) \in \mathcal{E}$, then for all $j<J,\left\{\left(W_{k}, \lambda_{j}^{-1 / 2} w_{k}\right)\right\}_{k \in I_{j}}$ is an orthonormal fusion basis for $E_{j}$, in particular $w_{k}^{2}=\lambda_{j}$ for $k \in I_{j}$.

Proof. By Theorem 3.1 (5) and Theorem 4.2, if $\mathbf{W}$ is a local minimizer of $F F P_{\mathbf{w}}$, then for all $j<J,\left\{W_{k}\right\}_{k \in I_{j}}$ is a Riesz fusion basis for $E_{j}$ and

$$
T_{j}^{*} T_{j}=\lambda_{j} I_{\oplus_{k \in I_{j}}} W_{k},
$$

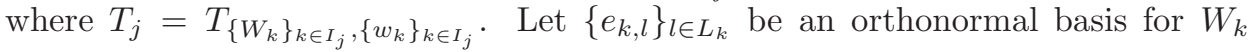
for all $k=1, \ldots, K$. Then $\left\{\left\{\left(\delta_{k, m} e_{k, l}\right)_{m \in I_{j}}\right\}_{l \in L_{k}}\right\}_{k \in I_{j}}$ is an orthonormal basis for $\bigoplus_{k \in I_{j}} W_{k}$. If $k, k^{\prime} \in I_{j}, l \in L_{k}$ and $l^{\prime} \in L_{k^{\prime}}$, then

$$
\begin{aligned}
\left\langle e_{k, l}, e_{k^{\prime}, l^{\prime}}\right\rangle & =\left\langle\frac{1}{w_{k}} T_{j}\left(\delta_{k, m} e_{k, l}\right)_{m \in I_{j}}, \frac{1}{w_{k^{\prime}}} T_{j}\left(\delta_{k^{\prime}, m} e_{k^{\prime}, l^{\prime}}\right)_{m \in I_{j}}\right\rangle \\
& =\frac{1}{w_{k} w_{k^{\prime}}}\left\langle\left(\delta_{k, m} e_{k, l}\right)_{m \in I_{j}}, T_{j}^{*} T_{j}\left(\delta_{k^{\prime}, m} e_{k^{\prime}, l^{\prime}}\right)_{m \in I_{j}}\right\rangle \\
& =\frac{\lambda_{j}}{w_{k} w_{k^{\prime}}}\left\langle\left(\delta_{k, m} e_{k, l}\right)_{m \in I_{j}},\left(\delta_{k^{\prime}, m} e_{k^{\prime}, l^{\prime}}\right)_{m \in I_{j}}\right\rangle=\frac{\lambda_{j}}{w_{k} w_{k^{\prime}}} \delta_{k, k^{\prime}} \delta_{l, l^{\prime}}
\end{aligned}
$$

This shows that the subspaces of the family $\left\{W_{k}\right\}_{k \in I_{j}}$ are orthogonal and $1=$ $\left\|e_{k, l}\right\|^{2}=\frac{\lambda_{j}}{w_{k}^{2}}$. Since also $\left\{W_{k}\right\}_{k \in I_{j}}$ is a Riesz fusion basis for $E_{j}$, the family $\left\{\left(W_{k}, \lambda_{j}^{-1 / 2} w_{k}\right)\right\}_{k \in I_{j}}$ is an orthonormal fusion basis for $E_{j}$.

\section{A CONCEPT OF IRREgUlarity AND THE STRUCTURE OF THE MiNimizers}

The next lemma leads to a notion of irregularity of a positive decreasing sequence, which is presented in [11] and extends the one in [5].

Lemma 5.1. Let $d \leq \sum_{k=1}^{K} L_{k}$. For any real positive decreasing sequence $\left\{c_{k}\right\}_{k=1}^{K}$, there exists a unique index $N_{0}$ with $1 \leq N_{0} \leq K$ such that the inequality

$$
\left(d-\sum_{k=1}^{j} L_{k}\right) c_{j}>\sum_{k=j+1}^{K} L_{k} c_{k}
$$

holds for $1 \leq j<N_{0}$, while

$$
\left(d-\sum_{k=1}^{j} L_{k}\right) c_{j} \leq \sum_{k=j+1}^{K} L_{k} c_{k}
$$

holds for $N_{0} \leq j \leq K$.

Proof. We will assume that any summation over an empty set is zero. Let $\mathcal{I}$ the set of indices such that (5.1) holds, which is non-empty since $K \in \mathcal{I}$. Let $j \in \mathcal{I}$. If $\sum_{k=1}^{j} L_{k}>d$ then it is immediate that $j+1 \in \mathcal{I}$. If $\sum_{k=1}^{j} L_{k} \leq d$ then

$$
\begin{aligned}
{\left[d-\sum_{k=1}^{j+1} L_{k}\right] c_{j+1} } & =-L_{j+1} c_{j+1}+\left(d-\sum_{k=1}^{j} L_{k}\right) c_{j+1} \leq-L_{j+1} c_{j+1}+\left(d-\sum_{k=1}^{j} L_{k}\right) c_{j} \\
& \leq-L_{j+1} c_{j+1}+\sum_{k=j+1}^{K} L_{k} c_{k}=\sum_{k=j+2}^{K} L_{k} c_{k}
\end{aligned}
$$

Therefore $k+1 \in \mathcal{I}$. Set $N_{0}$ as the minimum index of $\mathcal{I}$. 
We say that the $(\mathbf{L}, d)$-irregularity of a real nonnegative decreasing sequence $\left\{c_{k}\right\}_{k=1}^{K}$ is $N_{0}-1$, where $N_{0}$ is the unique index from the previous Lemma.

\subsection{The fundamental inequality.}

Definition 5.2. We say that $(\mathbf{w}, \mathbf{L})$ satisfies the fundamental inequality if

$$
\max _{k=1, \ldots, K} w_{k}^{2} \leq \frac{1}{d} \sum_{k=1}^{K} w_{k}^{2} L_{k} .
$$

Remark 5.3. If $(\mathbf{w}, \mathbf{L})$ satisfies the fundamental inequality, then

$$
\max _{k=1, \ldots, K} w_{k}^{2} \leq \frac{1}{d} \sum_{k=1}^{K} w_{k}^{2} L_{k} \leq \frac{1}{d} \max _{k=1, \ldots, K} w_{k}^{2} \sum_{k=1}^{K} L_{k},
$$

thus $\sum_{k=1}^{K} L_{k} \geq d$. Note that this restriction is obviously required for fusion frames to exist for that $\mathbf{L}$.

Remark 5.4. Let $w \in \mathbb{R}_{>0}$. Then $(w, \mathbf{L})$ satisfies the fundamental inequality if and only if $d \leq \sum_{k=1}^{K} L_{k}$. In particular, if $L \in \mathbb{N}$, then $(w, L)$ satisfies the fundamental inequality if and only if $d \leq K L$.

Proposition 5.5. If $(\mathbf{W}, \mathbf{w})$ is an $\alpha$-tight fusion frame for $\mathbb{F}^{d}$ with $\mathbf{W} \in \mathcal{S}_{K}(\mathbf{L})$ then $(\mathbf{w}, \mathbf{L})$ satisfies the fundamental inequality.

Proof. For $k \in\{1, \ldots, K\}$,

$$
\begin{aligned}
w_{k}^{2} L_{k} & =w_{k}^{2} \operatorname{tr}\left(\pi_{W_{k}}\right) \leq \sum_{k^{\prime}=1}^{K} w_{k^{\prime}}^{2} \operatorname{tr}\left(\pi_{W_{k^{\prime}}} \pi_{W_{k}}\right)=\operatorname{tr}\left(\sum_{k^{\prime}=1}^{K} w_{k^{\prime}}^{2} \pi_{W_{k^{\prime}}} \pi_{W_{k}}\right) \\
& =\operatorname{tr}\left(S_{\mathbf{W}, \mathbf{w}} \pi_{W_{k}}\right)=\operatorname{tr}\left(\alpha I_{\mathcal{H}} \pi_{W_{k}}\right)=\alpha \operatorname{tr}\left(\pi_{W_{k}}\right)=\alpha L_{k}
\end{aligned}
$$

Hence, $w_{k}^{2} \leq \alpha$ for all $k=1, \ldots, K$. On the other hand,

$$
\sum_{k=1}^{K} w_{k}^{2} L_{k}=\sum_{k=1}^{K} w_{k}^{2} \operatorname{tr}\left(\pi_{W_{k}}\right)=\operatorname{tr}\left(S_{\mathbf{W}, \mathbf{w}}\right)=\operatorname{tr}\left(\alpha I_{\mathcal{H}}\right)=\alpha d
$$

So the result follows.

The following lemma establishes the relation between the concept of irregularity and the fundamental inequality.

Lemma 5.6. Suppose that $\mathbf{w}$ is arranged in decreasing order. Then $(\mathbf{w}, \mathbf{L})$ satisfies the fundamental inequality if and only if the $(\mathbf{L}, d)$-irregularity of $\mathbf{w}$ is zero.

Proof. The pair $(\mathbf{w}, \mathbf{L})$ satisfies the fundamental inequality if and only if $w_{1}^{2} \leq$ $\frac{1}{d} \sum_{k=1}^{K} L_{k} w_{k}^{2}$, i.e., $\left(d-L_{1}\right) w_{1}^{2} \leq \sum_{k=2}^{K} L_{k} w_{k}^{2}$, and this is equivalent to say that the $(\mathbf{L}, d)$-irregularity of $\mathbf{w}^{2}$ is zero.

5.2. The concept of irregularity and the structure of the minimizers. To prove Theorem 5.9 we need the following proposition.

Proposition 5.7. Assume that $\mathbf{w}$ is arranged in decreasing order and that $\mathbf{W}$ is a local minimizer of $F F P_{\mathbf{w}}$, such that $(\mathbf{W}, \mathbf{w}) \in \mathcal{E}$. If $\mathbf{w}$ has $(\mathbf{L}, d)$-irregularity $N_{0}-1$, then $I_{J}=\left\{N_{0}, \ldots, K\right\}$. 
Proof. Reasoning as in [5], we will first show that $\left\{N_{0}, \ldots, K\right\} \subseteq I_{J}$, which is equivalent to prove that $I_{J}^{c} \subseteq\left\{1, \ldots, N_{0}-1\right\}$. Let $k \in I_{J}^{c}$. Using Theorem 3.1(1)(3)(4) and Theorem 4.3 .

$$
\begin{aligned}
\sum_{i=k+1}^{K} L_{i} w_{i}^{2} & =w_{k}^{2} \sum_{i=1, i \in I_{J}}^{k} L_{i}+\sum_{i=k+1}^{K} L_{i} w_{i}^{2}-w_{k}^{2} \sum_{i=1, i \in I_{J}}^{k} L_{i} \\
& \leq \sum_{i=1, i \in I_{J}}^{k} L_{i} w_{i}^{2}+\sum_{i=k+1}^{K} L_{i} w_{i}^{2}-w_{k}^{2} \sum_{i=1, i \in I_{J}}^{k} L_{i} \\
& \leq \sum_{i \in I_{J}} L_{i} w_{i}^{2}+\sum_{i=k+1, i \in I_{J}^{c}}^{K} L_{i} w_{i}^{2}-w_{k}^{2} \sum_{i=1, i \in I_{J}}^{k} L_{i} \\
& =\lambda_{J} \operatorname{dim}\left(E_{J}\right)+\sum_{i=k+1, i \in I_{J}^{c}}^{K} L_{i} w_{i}^{2}-w_{k}^{2} \sum_{i=1, i \in I_{J}}^{k} L_{i} \\
& =\lambda_{J}\left(d-\sum_{j=1}^{J-1} \sum_{k \in I_{j}} L_{k}\right)+\sum_{i=k+1, i \in I_{J}^{c}}^{K} L_{i} w_{i}^{2}-w_{k}^{2} \sum_{i=1, i \in I_{J}}^{k} L_{i} \\
& <\left(d-\sum_{k \in I_{J}^{c}} L_{k}+\sum_{i=k+1, i \in I_{J}^{c}}^{K} L_{i}-\sum_{i=1, i \in I_{J}}^{k} L_{i}\right) w_{k}^{2} \\
& =\left(d-\sum_{i=1}^{k} L_{i}\right) w_{k}^{2} .
\end{aligned}
$$

Hence $k \in\left\{1, \ldots, N_{0}-1\right\}$.

To see that $I_{J} \subseteq\left\{N_{0}, \ldots, K\right\}$ it is only left to prove that $\left\{1, \ldots, N_{0}-1\right\} \cap I_{J}=\emptyset$. Assume to the contrary that $\left\{1, \ldots, N_{0}-1\right\} \cap I_{J} \neq \emptyset$. Let $k_{0}=\min \left\{1, \ldots, N_{0}-\right.$ $1\} \cap I_{J}$ and $k_{1}=\max \left\{1, \ldots, N_{0}-1\right\} \cap I_{J}$. We have $k_{0}=\min I_{J}$, so $\left\{k_{0}\right\}=$ $\left\{1, \ldots, k_{0}\right\} \cap I_{J}$. Thus, by Theorem $3.1(5)$ and Proposition 5.5.

$$
\begin{aligned}
w_{k_{0}}^{2}=\max _{k \in I_{J}} w_{k}^{2} & \leq \frac{1}{\operatorname{dim}\left(E_{J}\right)} \sum_{k \in I_{J}} L_{k} w_{k}^{2} \\
\left(\operatorname{dim}\left(E_{J}\right)-L_{k_{0}}\right) w_{k_{0}}^{2} & \leq \sum_{k \in I_{J}, k>k_{0}} L_{k} w_{k}^{2} \\
\left(\operatorname{dim}\left(E_{J}\right)-\sum_{k \in\left\{1, \ldots, k_{0}\right\} \cap I_{J}} L_{k}\right) w_{k_{0}}^{2} & \leq \sum_{k \in I_{J}, k>k_{0}} L_{k} w_{k}^{2} .
\end{aligned}
$$

Hence, by Theorem 3.1(1)(3) and Theorem 4.3 .

$$
\begin{aligned}
\left(d-\sum_{k \in I_{J}^{c}} L_{k}-\sum_{k \in\left\{1, \ldots, k_{1}\right\} \cap I_{J}} L_{k}\right) w_{k_{1}}^{2} & =\left(d-\sum_{j=1}^{J-1} \sum_{k \in I_{j}} L_{k}-\sum_{k \in\left\{1, \ldots, k_{1}\right\} \cap I_{J}} L_{k}\right) w_{k_{1}}^{2} \\
& =\left(\operatorname{dim}\left(E_{J}\right)-\sum_{k \in\left\{1, \ldots, k_{1}\right\} \cap I_{J}} L_{k}\right) w_{k_{1}}^{2} \\
& \leq \sum_{k \in I_{J}, k>k_{1}} L_{k} w_{k}^{2} .
\end{aligned}
$$


Since $\left\{N_{0}, \ldots, K\right\} \subseteq I_{J}$ and $k_{1}$ is the maximum of $\left\{1, \ldots, N_{0}-1\right\} \cap I_{J}$, we have $\left\{1, \ldots, N_{0}-1\right\}=I_{J}^{c} \cup\left(\left\{1, \ldots, k_{1}\right\} \cap I_{J}\right)$, and thus, $d-\sum_{k=1}^{N_{0}-1} L_{k}=d-\sum_{k \in I_{J}^{c}} L_{k}-$ $\sum_{k \in\left\{1, \ldots, k_{1}\right\} \cap I_{J}} L_{k}$. Also, $\left\{k_{1}+1, \ldots, K\right\} \cap I_{J}=\left\{N_{0}, \ldots, K\right\}$.

Therefore, from the previous inequality, $\left(d-\sum_{k=1}^{N_{0}-1} L_{k}\right) w_{N_{0}-1}^{2} \leq \sum_{k=N_{0}}^{K} L_{k} w_{k}^{2}$. But the definition of the irregularity $N_{0}$ gives that $\left(d-\sum_{i=1}^{k} L_{i}\right) w_{k}^{2}>\sum_{i=k+1}^{K} L_{i} w_{i}^{2}$ for all $k<N_{0}$, which is a contradiction for $k=N_{0}-1$.

If $\mathbf{W}$ is a local minimizer of $F F P_{\mathbf{w}}$ such that $(\mathbf{W}, \mathbf{w}) \in \mathcal{E}$, then Proposition 5.7 shows that $I_{J} \neq \emptyset$. So we can state the following:

Corollary 5.8. If $\mathbf{W}$ is a local minimizer of $F F P_{\mathbf{w}}$ such that $(\mathbf{W}, \mathbf{w}) \in \mathcal{E}$, then $(\mathbf{W}, \mathbf{w})$ is a fusion frame.

Theorem 5.9. Let $\mathbf{L} \in \mathbb{N}^{K}$ and $\mathbf{w}$ be a vector of decreasing weights with $(\mathbf{L}, d)$ irregularity equal to $N_{0}$. Then any local minimizer $\mathbf{W}$ of the frame potential $F F P_{\mathbf{w}}$ such that $(\mathbf{W}, \mathbf{w}) \in \mathcal{E}$, can be decomposed as

$$
(\mathbf{W}, \mathbf{w})=\left\{\left(W_{k}, w_{k}\right)\right\}_{k=1}^{N_{0}-1} \cup\left\{\left(W_{k}, w_{k}\right)\right\}_{k=N_{0}}^{K},
$$

where $\left\{\left(W_{k}, \lambda_{j}^{-1 / 2} w_{k}\right)\right\}_{k=1}^{N_{0}-1}$ is an orthonormal fusion frame sequence for whose orthogonal complement the sequence $\left\{\left(W_{k}, w_{k}\right)\right\}_{k=N_{0}}^{K}$ is a tight fusion frame.

Proof. It follows from Theorem 3.1, Theorem4.3, Proposition 5.7 and Corollary 5.8

Corollary 5.10. Let $\mathbf{L} \in \mathbb{N}^{K}$ and $\mathbf{w}$ be a vector of decreasing weights with $(\mathbf{L}, d)$ irregularity equal to $N_{0}$. Then any local minimizer of the fusion frame potential $F F P_{\mathbf{w}}$, which belongs to $\mathcal{E}$, is also a global minimizer and the minimum value is

$$
\sum_{k=1}^{N_{0}-1} w_{k}^{4} L_{k}+\frac{1}{d-\sum_{k=1}^{N_{0}-1} L_{k}}\left(\sum_{k=N_{0}}^{K} w_{k}^{2} L_{k}\right)^{2} .
$$

Proof. Let $\mathbf{W}$ be any local minimizer in $\mathcal{E}$. Then we have the decomposition of Theorem 5.9. On the one hand, $\pi_{W_{k}} \pi_{W_{k^{\prime}}}=0$ for $k^{\prime} \neq k=1, \ldots, N_{0}-1$, and hence

$$
\sum_{k, k^{\prime}=1}^{N_{0}-1} w_{k}^{2} w_{k^{\prime}}^{2} \operatorname{tr}\left(\pi_{W_{k}} \pi_{W_{k^{\prime}}}\right)=\sum_{k=1}^{N_{0}-1} w_{k}^{4} L_{k}
$$

On the other hand, $\operatorname{dim}\left(\operatorname{span} \bigcup_{k=1}^{N_{0}-1} W_{k}\right)=\sum_{k=1}^{N_{0}-1} L_{k}$, so $\operatorname{dim}\left(\operatorname{span} \bigcup_{k=N_{0}}^{K} W_{k}\right)=$ $d-\sum_{k=1}^{N_{0}-1} L_{k}$ and by Proposition 2.4.

$$
\sum_{k, k^{\prime}=N_{0}}^{K} w_{k}^{2} w_{k \prime}^{2} \operatorname{tr}\left(\pi_{W_{k}} \pi_{W_{k^{\prime}}}\right)=\frac{1}{d-\sum_{k=1}^{N_{0}-1} L_{k}}\left(\sum_{k=N_{0}}^{K} w_{k}^{2} L_{k}\right)^{2} .
$$

Therefore

$$
F F P_{\mathbf{w}}(\mathbf{W}, \mathbf{w})=\sum_{k=1}^{N_{0}-1} w_{k}^{4} L_{k}+\frac{1}{d-\sum_{k=1}^{N_{0}-1} L_{k}}\left(\sum_{k=N_{0}}^{K} w_{k}^{2} L_{k}\right)^{2} .
$$

We have proved that every local minimizer in $\mathcal{E}$ of the fusion frame potential attains the same value (5.3). By Proposition 4.1.2 of [11], (5.3) is a lower bound for $F F P_{\mathbf{w}}$. Hence every local minimizer is also a global minimizer and it attains the value given in (5.3) 
Remark 5.11. It can be seen that if a fusion frame $(\mathbf{W}, \mathbf{w})$ has the structure described in Theorem [5.9, then $(\mathbf{W}, \mathbf{w}) \in \mathcal{E}$. In [11, Proposition 4.1.2] it is shown that $(\mathbf{W}, \mathbf{w})$ has the structure described in Theorem 5.9 if and only if the value given in (5.3) is attained.

\section{Existence OF Tight FUSION FRAMES}

Tight fusion frames, as well as tight frames, are particular useful mainly because they allow a very simple and computational efficient representation of the elements of $\mathcal{H}$. In this section we relate the the existence of tight fusion frames with the minimizers of the fusion frame potential and the fundamental inequality.

Proposition 6.1. Let $(\mathbf{w}, \mathbf{L})$ satisfy the fundamental inequality and let $\mathbf{W}$ be a local minimizer of the fusion frame potential $F F P_{\mathbf{w}}$, such that $(\mathbf{W}, \mathbf{w}) \in \mathcal{E}$. Then $(\mathbf{W}, \mathbf{w})$ is a tight fusion frame.

Proof. Let $(\mathbf{w}, \mathbf{L})$ satisfy the fundamental inequality and $\mathbf{W}$ be a local minimizer of FFP $\mathbf{w}_{\mathbf{w}}$. By Theorem 3.1 and Theorem 4.3, if $(\mathbf{W}, \mathbf{w})$ is not tight, then $J>1$ and

$$
\begin{aligned}
\sum_{k=1}^{K} w_{k}^{2} L_{k} & =\sum_{j=1}^{J-1} \sum_{k \in I_{j}} w_{k}^{2} L_{k}+\sum_{k \in I_{J}} w_{k}^{2} L_{k}=\sum_{j=1}^{J-1} \sum_{k \in I_{j}} \lambda_{j} L_{k}+\lambda_{J} \operatorname{dim}\left(E_{J}\right) \\
& <\lambda_{1} \sum_{j=1}^{J-1} \sum_{k \in I_{j}} L_{k}+\lambda_{1} \operatorname{dim}\left(E_{J}\right)=\lambda_{1}\left(\sum_{j=1}^{J-1} \operatorname{dim}\left(E_{j}\right)+\operatorname{dim}\left(E_{J}\right)\right) \\
& =\lambda_{1}\left(d-\operatorname{dim}\left(E_{J}\right)+\operatorname{dim}\left(E_{J}\right)\right)=w_{1}^{2} d,
\end{aligned}
$$

which contradicts that $(\mathbf{w}, \mathbf{L})$ satisfies the fundamental inequality. Therefore, $(\mathbf{W}, \mathbf{w})$ is a tight fusion frame.

We will consider the following class of pairs of weights and dimensions:

$\mathcal{M}=\{(\mathbf{w}, \mathbf{L})$ : there exists a local minimizer of the frame potential

$$
\left.F F P_{\mathbf{w}} \text { that belongs to } \mathcal{E}\right\} \text {. }
$$

As a consequence of Proposition 5.5 and Proposition 6.1 we obtain:

Theorem 6.2. Assume that $(\mathbf{w}, \mathbf{L}) \in \mathcal{M}$ There exists a tight fusion frame $(\mathbf{W}, \mathbf{w})$ for $\mathbb{F}^{d}$ with $\mathbf{W} \in \mathcal{S}_{K}(\mathbf{L})$ if and only if $(\mathbf{w}, \mathbf{L})$ satisfies the fundamental inequality.

In [1] a characterization is given under the restriction that $\sum_{k=1}^{K} w_{k}^{2} L_{k}=1$, in the form of Horn-Klyachko compatibility conditions. It can be complicated to use in practice, since it involves the computation of the Littlewood-Richardson coefficients of certain associated partitions.

Remark 6.3. The previous theorem can be used to show that some pairs $(\mathbf{w}, \mathbf{L})$ are not in $\mathcal{M}$. For instance, consider $d=3, K=L=2$ and $w_{1}=w_{2}=1$. In this case $d \leq K L$. We are going to see that $(1,2) \notin \mathcal{M}$. Assume by contradiction that $(1,2) \in \mathcal{M}$. By Theorem 6.2. there exists a tight fusion frame with fusion frame bounds $\frac{K L}{d}=\frac{4}{3}$. Let $P_{1}, P_{2}$ be orthogonal projections such that

$$
\frac{4}{3} I=P_{1}+P_{2} \text {. }
$$


Let $U_{1}$ be a unitary matrix and let $D_{1}$ a diagonal matrix such that $P_{1}=U_{1} D_{1} U_{1}^{*}$. From (6.1), $\frac{4}{3} I=D_{1}+U_{1}^{*} P_{2} U_{1}$ where $D_{1}$ and $U_{1}^{*} P_{2} U_{1}$ are orthogonal projections. The elements of the diagonal satisfy that $\alpha^{2}=\alpha$, therefore $\alpha=0$ or $\alpha=1$. Since, $\operatorname{tr}\left(D_{1}\right)=\operatorname{tr}\left(P_{1}\right)=2$ we can assume $D_{1}=\left(\begin{array}{lll}1 & 0 & 0 \\ 0 & 1 & 0 \\ 0 & 0 & 0\end{array}\right)$. Thus, $U_{1}^{*} P_{2} U_{1}=\left(\begin{array}{ccc}\frac{1}{3} & 0 & 0 \\ 0 & \frac{1}{3} & 0 \\ 0 & 0 & \frac{4}{3}\end{array}\right)$ which is not idempotent, and hence it is not an orthogonal projection.

Remark 6.4. A frame can be seen as a fusion frame where all the subspaces are one-dimensional and the weights are equal to the norms of the elements of the frame. We note that in this case (5.2) coincides with the fundamental inequality for frames introduced in [5, where it has been proved that $(\mathbf{w}, 1)$ always belongs to $\mathcal{M}$. Thus Theorem 6.2 shows that the fundamental inequality (5.2) that involves the weights and the dimensions of the subspaces of fusion frames plays the same role as the one that involves the norms of frames.

If $\sum_{k=1}^{K} L_{k}=d$, set $\mathbf{W}$ such that $\mathbb{F}^{d}$ is the orthogonal sum of its elements. Clearly, $(\mathbf{W}, \mathbf{w}) \in \mathcal{E}$ and $F F P_{\mathbf{w}}(\mathbf{W}, \mathbf{w})=\sum_{k=1}^{K} w_{k}^{4} L_{k}$. If $w_{1}=\ldots=w_{K}$, then the $(\mathbf{L}, d)$-irregularity of $\mathbf{w}$ is 0 . If $N_{0}$ is such that $w_{1} \geq \ldots \geq w_{N_{0}-1}>w_{N_{0}}=$ $\ldots=w_{K}$, then the $(\mathbf{L}, d)$-irregularity of $\mathbf{w}$ is $N_{0}-1$. In both cases, the value of $F F P_{\mathbf{w}}(\mathbf{W}, \mathbf{w})$ coincides with (5.3), so, by [11, Proposition 4.1.2], $\mathbf{W}$ is a global minimizer. Therefore, if $\sum_{k=1}^{K} L_{k}=d$, the pair $(\mathbf{w}, \mathbf{L}) \in \mathcal{M}$ for any $\mathbf{w}$.

\section{REFERENCES}

[1] J.J. Benedetto, M. Fickus. Finite normalized tight frames, Adv. Comput. Math., 18(2-4):357$385,2003$.

[2] I. Carrizo, S. Heineken. Critical pairs of sequences of a mixed frame potential, Numerical Functional Analysis and Optimization, 35: 665-684, 2014.

[3] P. G. Casazza. The art of frame theory, Taiwanese Jour. of Math., 4(2):129-202, 2000.

[4] P.G. Casazza, M. Fickus. Minimizing fusion frame potential, Acta Appl. Math., 107(1-3): 7-24, 2009.

[5] P.G. Casazza, M. Fickus, J. Kovacevic, M. T. Leon, J. C. Tremain. A physical interpretation of tight frames. In: Harmonic Analysis and Applications. Appl. Numer. Harmon. Anal., pp. 51-76, , Birkhäuser Boston, Boston, 2006.

[6] P. G. Casazza and G. Kutyniok Eds., Finite Frames. Theory and Applications, Birkhäuser, Boston, 2012.

[7] P. G. Casazza and G. Kutyniok. Frames of subspaces, Contemp. Math., 345:87-113, 2004.

[8] P. G. Casazza, G. Kutyniok and S. Li. Fusion frames and distributed processing, Appl. Comput. Harmon. Anal., 25:114-132, 2008.

[9] O. Christensen, An introduction to frames and Riesz bases, Birkhäuser, Boston, 2003.

[10] J. Kovačević and A. Chebira. An introduction to frames, Found. Trends Signal Process., 2:1-94, 2008.

[11] P.G. Massey, M. A. Ruiz, D. Stojanoff. The structure of the minimizers of the frame potential on fusion frames, J. Fourier Anal. Appl., 16:514-543, 2010. 